\title{
Exploring Symmetries in Breast MRI Scan
}

\author{
Robert Alterson and Donald B. Plewes \\ Department of Medical Imaging, University of Toronto, 2075 Bayview Avenue, Toronto, \\ Ontario, M4N 3M5 Canada \\ rsalsten. sunnybrook.utoronto.ca \\ http://www.swchsc.on.ca/ dbp/mainfr.html
}

\begin{abstract}
In this work we employ computer-vision techniques to detect natural biological symmetries in breast MR scans. Currently, breast MR images are assessed in terms of the kinetics and location of uptake of Gd-DTPA. However, mammographic interpretation often uses symmetry between left and right breasts to indicate the site of potential tumour masses but has not been used in breast MRI. In this study, we present such a method for characterizing breast symmetry based on three objective measures of similarity including multiresolution non-orthogonal wavelet representation, three-dimensional intensity distributions and co-occurrence matrices. Statistical feature distributions that are invariant to feature localization are computed for each of the similarity metrics. These distributions are later compared against each other to account for perceptual similarity. Studies based on 51 normal MRI scans of randomly selected patients showed that the sensitivity of symmetry detection rate approached 94\%. The symmetry analysis procedure presented in this paper can be applied as an aid in detecting breast tissue changes arising from disease.
\end{abstract}

\section{Introduction}

Symmetry between breasts has been used for many years in X-ray mammography to aid in the detection of disease. Typically, suspicious areas in one breast are compared, based on human visual perception, with the same area in the opposite breast and any differences may be reflective of disease processes. However, the use of symmetry in mammography is fundamentally limited by the fact that mammography is a 2D projection imaging method. Alternatively, MRI breast imaging offers several advantages, as it is a $3 \mathrm{D}$ imaging method that exhibits very high contrast between normal breast tissue entities. Currently, breast MRI is based on the use of Gd-DTPA enhancement reflective of tumour angiogenesis but virtually no use is made of breast symmetry in image interpretation.

In this work, we explore the question of symmetry between a patient's left and right breast in three-dimensional MRI scans. The purpose of this study is to verify that symmetry, in terms of volume, texture or structure, could be demonstrated in 3D breast MRI scans. Another objective is to develop a set of similarity metrics that can be used for longitudinal studies and for computer aided tracking of changes in breast tissue.

The research literature contains a number of studies that deal with breast symmetry analysis in the context of mammography. However, the question of breast symmetry 
analysis in MRI has not yet been addressed. There are several unique issues to breast MRI that differentiate this problem, as follows. Standard mammography compounds, by means of projection, an entire breast volume into one $2 \mathrm{D}$ plane. Conversely, MRI is a three-dimensional imaging modality that provides a true rendering of selected breast planes. Symmetry analysis must therefore be extended to capture symmetry properties over a range of imaged MRI slices. Further, due to imaging coil properties, MR images vary in intensity from one slice to the next and also between bilateral breasts imaged simultaneously.

The common approach to mammogram breast symmetry analysis involves coregistration of the two breasts followed by bilateral subtraction. Yin et al presented a method for automated analysis of bilateral mammograms [1]. Their method uses the anterior portions of the breast border, together with the nipple as landmarks for least square matching registration followed by nonlinear bilateral subtraction. Sallam and Bowyer performed analysis of bilateral mammograms by using registration followed by subtraction [2]. Registration was carried out using steerable filters, which are invariant to rotation and shift. Vujovic and Brazkovic describe a method for automatic extraction of registration landmarks using crossings of horizontal and vertical structures [3]. Their method is used to compare mammograms of the same breast taken at different times.

Registration of the same breast imaged at different times can be carried out with relative effectiveness. However, this approach may encounter problems when used for bilateral symmetry analysis for several reasons, as follows. Breasts have natural asymmetries in terms of size, shape and structure that make bilateral co-registration especially challenging. In addition, finding quality registration landmarks can often prove very difficult.

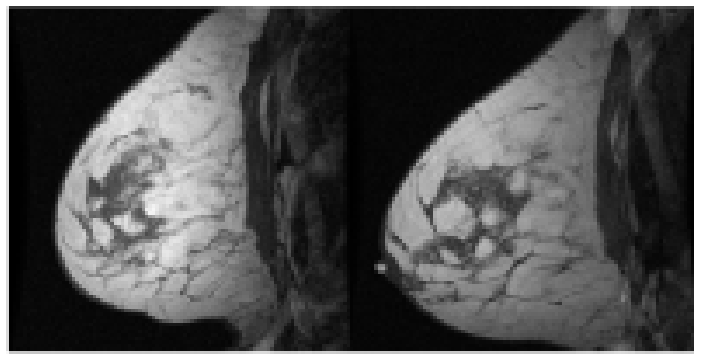

Fig. 1. Left and right breast MRI scans of the same patient appear similar by visual observation. However, standard image comparison methods would perform poorly in this case. Methods that take into account the structure and texture must be therefore applied.

\section{Method}

To assess breast symmetry, we employed objective similarity measures that are based on theoretical models for image comparison. In order to effectively compare threedimensional MRI breast scans, effective feature extraction methods must be selected 
and applied. As mentioned earlier, direct comparison methods that are based on linear preprocessing followed by image subtractions cannot be utilized here because of potential significant registration errors. The registration difficulty is compounded by the three dimensional nature of MRI breast scans. In other words, while mammographic image comparison requires the registration of two bilateral X-ray scans, MRI comparison would require the registration of two bilateral sets of images. MRI volumes can contain tens of images per breast. Therefore, features that represent image qualities with no localization dependency were chosen for this study.

One of the image feature sets selected are non-orthogonal Gabor wavelets, which are used for texture characterization [4]. In addition, we used co-occurrence matrices combined with cumulative intensity distributions to represent volumes of fiberglandular tissue and fat [5]. Prior to feature extraction we apply intensity normalization to correct for intensity variations. In addition, we use segmentation to separate breasts from the background.

\subsection{Non-orthogonal Gabor Wavelets}

One-dimensional Gabor analysis was originally introduced as a means for optimal joint time/frequency analysis of transmitted signals in communications systems. This theory was later extended into two dimensions and applied to problems in computer vision. Gabor wavelets also have biological counterparts in the cortical human visual systems as they can effectively capture orientation and spatial frequency qualities [6].

Some of the more prominent applications of Gabor wavelets are in the area of texture characterization. Texture plays a key role in human vision, and has an important part in the determination of objects' shape. The visual cortex uses texture to establish the shape of three-dimensional bodies. In fact, the reason high-end computer graphics and visualization systems require higher resolution is in order to support increased texture display requirements.

Gabor wavelets can efficiently localize frequency and orientation properties of an analyzed image. Furthermore, results of texture segmentation experiments using Gabor wavelets have produced strong correlation with results of identical segmentation tasks performed by humans [7]. Gabor wavelets have been applied to many areas of computer vision. These include texture segmentation, document analysis, edge detection, retina identification, target detection, fractal dimension measurement, image coding, and image representation [8].

The general form of the complex Gabor wavelet function is presented below [9]. It consists of a two-dimensional Gaussian function with standard deviation $\sigma$ that modulates a spatial sinusoid as follows:

$$
G_{i}(x, y)=e^{-\frac{x^{2}+y^{2}}{2 \sigma^{2}}} e^{j\left(\omega_{x} x+\omega_{y} y\right)}=e^{-\frac{\vec{v} \cdot \vec{v}}{2 \sigma^{2}}} e^{j \bar{\omega} \vec{v}}
$$

where $\omega_{x}$ and $\omega_{y}$ represent the spatial frequencies in the horizontal and vertical directions, respectively. By convolving a Gabor function $G$ with image $I$ we obtain the image $W$ as follows: 


$$
W(x, y)=\iint I(u, v) \cdot G(x-u, y-v) d u d v
$$

Fourier analysis of the general Gabor function is presented below.

$$
G\left(u_{x}, u_{y}\right)=2 \pi \sigma^{2} e^{-\frac{\left(u_{x}-\omega_{x}\right)^{2}+\left(u_{y}-\omega_{y}\right)^{2}}{2} \sigma^{2}}
$$

In the Fourier domain, the general Gabor wavelet function consists of a shifted Gaussian with a displacement proportional to the central frequency parameter of the base function. Its width, however, is determined by $1 / \sigma$ and is thus inversely proportional to its spatial domain width. Notice that the Gaussian localization property is preserved by the Gabor function, i.e., there is an optimal trade-off in localization for both frequency and spatial domains.

The standard deviation value for Gabor wavelet base functions can be determined according to the desired frequency and angular frequency-domain bandwidths. Expression 4 provides the relation between the frequency bandwidth, $B_{f}$, in octaves and the spatial standard deviation, $\sigma . f$ represents the selected central frequency and the cut-off points are set to $-6 \mathrm{db}$. A cut-off point is defined as the location where the wavelet amplitude reaches a value that is equal to half of its peak magnitude.

$$
\begin{gathered}
\sigma=\frac{\sqrt{\ln (2) / 2}}{f \pi} \cdot \frac{2^{B_{f}}+1}{2^{B_{f}}-1} \\
\sigma=\frac{\sqrt{\ln (2) / 2}}{f \pi} \cdot \frac{1}{\tan \left(B_{\theta} / 2\right)}
\end{gathered}
$$

Expression 5 quantifies the Gabor function's standard deviation as a function of the desired angular bandwidth, $B_{\theta}$. Similarly, the cut-off points are also set here to -6 db. By dividing Expression 5 by Expression 4 we can derive the relationship between the frequency and angular bandwidths.

To summarize, Gabor wavelets can be efficiently used for texture segmentation. However, determining optimal values for the wavelet parameters is not a trivial problem. The wavelet parameters have to be selected in such way that textural properties are properly captured. The central frequency and orientation together with their respective bandwidths must lie close to the dominant texture components of interest. To illustrate this, Figure 2 depicts the application of Gabor wavelets to a single MR breast scan.

\subsection{Co-occurrence Matrices}

The gray level co-occurrence matrix (GLCM) representation is a well-studied method used for texture characterization. This method relies on second order statistics via es- 
timation of the second order joint probability distribution. Ohanian and Dubes [10] demonstrated the high viability of this method for extraction of texture features.

Co-occurrence matrices can be constructed in eight different spatial directions and for a number of distances as follows:

$$
C M, \Phi(d, \theta)=[\hat{f}(i, j \mid d, \theta)]
$$

where $d$ and $\theta$ represent the displacement and angle of separation, respectively. The elements of the co-occurrence matrix represent the probability that two image elements at a given separation would have values equal to $i$ and $j$. The co-occurrence matrix is always square but only rarely symmetric.

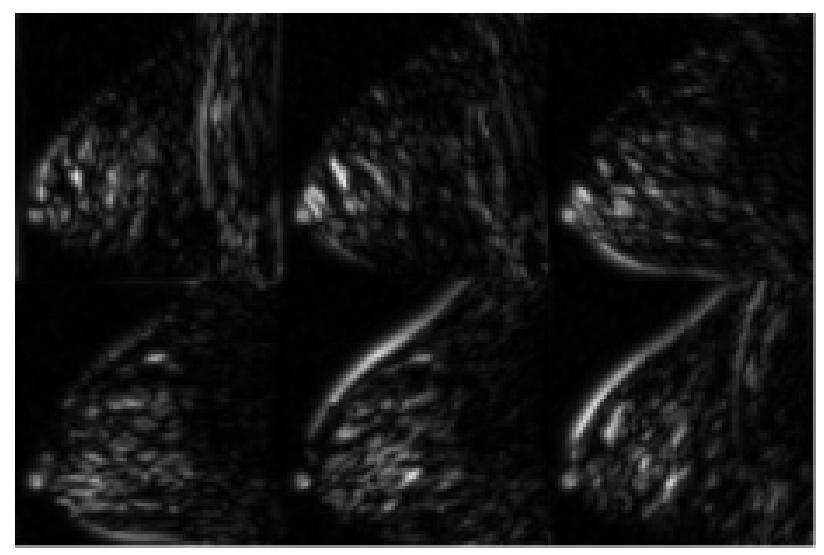

Fig. 2. This figure depicts the magnitude response of Gabor wavelets as applied to the right breast scan in Figure 1. Six Gabor orientations at $\pi / 6$ angular spacing were used here to demonstrate the wavelet response at different orientations. The actual number of orientations used in this work was 18 with a bandwidth of 1 Octave and over 5 scales.

\subsection{Extracted Feature Comparison}

Once image features are extracted from each three-dimensional breast scan, we compute statistical feature distributions for the three similarity metrics selected. As discusses earlier, these distributions are invariant to feature localization as they contain global statistics for an entire breast volume. In order to effectively compare these statistical data, several metrics were evaluated in the context of three dimensional breast MRI scans, including the Chomski norm, Bhattacharyya distance, Matusita distance and the Divergence [11]. The Matusita distance is presented below,

$$
M\left(P_{L}, P_{R}\right)=\sqrt{\int\left(\sqrt{P_{L}}-\sqrt{P_{R}}\right)^{2}}
$$

where $M\left(P_{L}, P_{R}\right)$ represents the Matusita distance between the distributions $P_{L}$ and $P_{R}$, corresponding to a left and right breast MRI scans, respectively. 
The Matusita distance metric faired significantly better when compared with other distance metrics and was subsequently used for computing the results presented in this study.

\subsection{Patient Symmetry Evaluation}

For evaluation purposes we randomly selected 51 normal patients who had previously obtained bi-lateral breast MRI from a large population screening study. T1-weighted,

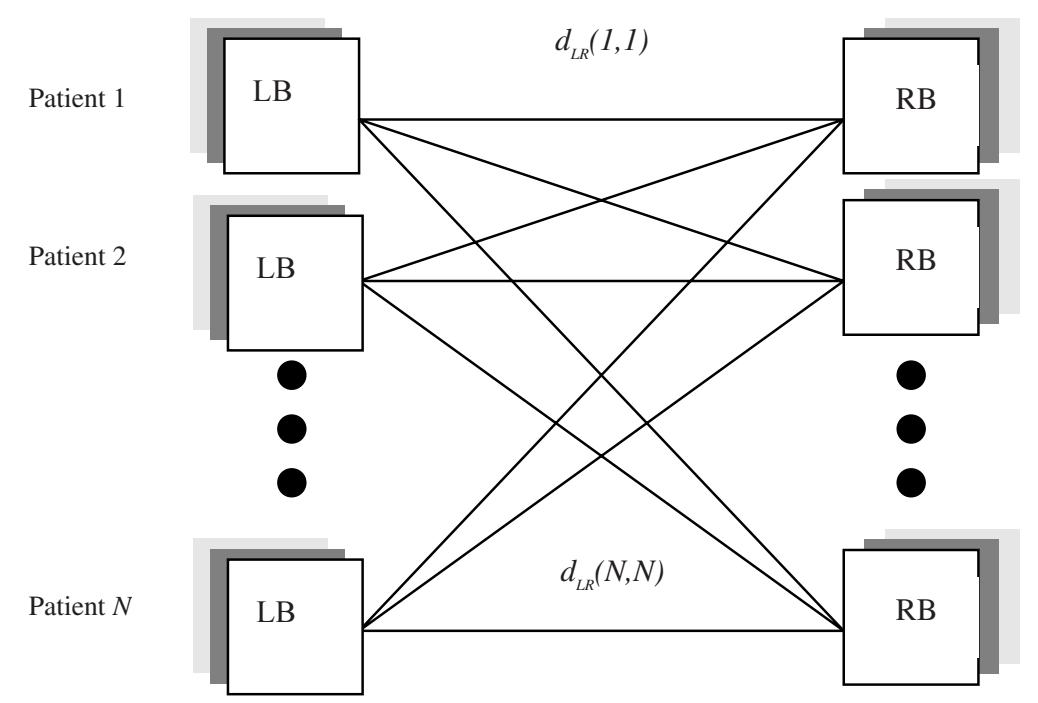

Fig. 3. An illustration of the comparison method applied for evaluation of the symmetry properties. Statistical feature properties of MRI volumes corresponding to left breasts are compared against the right breasts of the evaluation population. This process is repeated for the right breast volumes.

sagital images obtained prior to contrast enhancement by Gd-DTPA were used in the analysis. The three feature extraction techniques described earlier were used to produce statistics pertaining to the degree of symmetry detected between a breast and those from the entire population including the patient's contra-lateral breast. Figure 3 illustrates the comparison procedure for a given test population. The extracted features from each breast scan are compared, using the Matusita distance, which was described earlier, with the features of each of the contra-lateral breasts of the entire test population. The distance between two breast scans is denoted by $d_{L R}\left(L_{i}, R_{j}\right)=d_{R L}\left(R_{j}, L_{i}\right)$, where $R$ and $L$ represent right and left breast MRI scans with indices $i, j \in 1,2, . ., N$ and with $N$ equal to the size of the evaluation population or the number of patients. Note that due to the properties of the Matusita metric, the left-right feature distance between two breast scans is equal to the rightleft distance i.e., the distance metric is symmetric. 
Two types of breast symmetry were identified in this study. Full symmetry is defined when among the test population; both breasts of an analyzed patient are closest to their respective contra-lateral breast. Partial symmetry occurs when only one breast is closest to its contra-lateral breast.

\section{Results}

We computed symmetry rates obtained for each of the previously described featureextraction techniques. In addition, symmetry results based on a combination of the three feature classes are also presented. Detection rates for each of the comparison methods as well as the combined results are presented in table 1 (the closest match group threshold was set to the closest 4 patients or roughly $8 \%$ ). We obtained a detection rate of the patient's contra-lateral breast with a combined sensitivity approaching $94 \%$.

Table 1. Similarity data calculated with a threshold of less than $8 \%$ of the test population (51 patients population size). The high effectiveness of Gabor wavelets is evident, however, further improvement is achieved by combing it with 3D intensity distribution data and co-occurrence matrices.

\begin{tabular}{|l|l|l|}
\hline & Two-breast symmetry & One-breast symmetry \\
\hline $\begin{array}{l}\text { Intensity distributions } \\
\text { Co-occurrence matri- } \\
\text { ces }\end{array}$ & $62 \%$ & $76 \%$ \\
Gabor wavelets & $84 \%$ & $78 \%$ \\
Combined & $88 \%$ & $94 \%$ \\
\hline
\end{tabular}

As expected, texture analysis based on Gabor wavelets yielded the best results. This can be explained by the close resemblance these wavelets bear with the receptive field structures of cortical visual cells.

\section{Discussion}

This paper presents an effective procedure for evaluating the symmetry of contralateral, three-dimensional, MRI breast scans. For that purpose, several image features and feature comparison techniques are evaluated and compared. In that context, we have empirically selected optimal parameters for Gabor wavelet analysis of breast texture. The results obtained demonstrate that automated symmetry detection of breast symmetry is both feasible and accurate. While preliminary, these data suggest that improved detection accuracy may be possible beyond standard means to breast MRI interpretation. 
Our studies indicate that Gabor wavelets are superior to other feature extraction methods in terms of breast texture characterization. However, combining these features with others can improve accuracy.

The method presented can be used for longitudinal studies by applying it to breast MRI scans taken at different times. More specifically, a separate data file can be appended to each breast MR scan. These stored features can be used to track abnormal changes between successive scans of the same breast. Any changes in breast tissue due to a disease process will affect its texture. In turn, this discrepancy will cause the value of the comparison metric to change.

An issue to be addressed, however, is the effective partitioning of MRI breast scans into three-dimensional regions of interest (ROI). This will allow a more accurate localization of suspicious areas. To achieve this, alignment of contra lateral breasts would be required. This task may be carried out by comparison of Gabor wavelet features, computed over multiple scales.

\section{References}

1. F. F. Yin, M. L.Giger, K. Doi, C.J. Vyborny, and R.A. Schmidt, "Computerized detection of masses in digital mammograms: Automated alignment of breast images and its effect on bilateral-subtraction techniques", Medical Physics, vol. 21, no.3, pp.445-452, 1994.

2. M. Y. Sallam and K. W. Bowyer, Registration and difference analysis of corresponding mammogram images, Medical Image Analysis, Vol.3 No. 2, pp.103-118, 1999.

3. N. Vujovic and D. Brazkovic, "Establishing the correspondence between control points in pairs of mammographic images", IEEE Transactions Image Processing, vol. 6, pp.13881399, October 1997.

4. D. Dunn, W.E. Higgins, and J. Wakeley, "Texture segmentation using 2-D Gabor elementary functions", IEEE Trans. Pattern Analysis and Machine Intelligence, vol. 16, pp. 130-149, February 1994.

5. A. Pitiot, A.W. Toga, N. Ayache and P.M. Thompson, "Texture-Based MRI Segmentation with a Two-Stage Hybrid Neural Classifier", IEEE 2002 World Congress on Computational Intelligence and Neural Nets, Honolulu, HI, May 12-17, 2002.

6. P. Jones and L.A. Palmer, "An evaluation of the two-dimensional Gabor model of simple receptive fields in cat striate cortex", Journal of Neurophysiology, vol. 58, no. 6, pp. 1187-1211, December 1987.

7. J.R. Smith, Integrated Spatial and Feature Image Systems: Retrieval, Analysis and Compression, PhD dissertation, Graduate School of Arts and Sciences, Columbia University, 1997.

8. T.P. Weldon, W.E. Higgins, and D.F. Dunn, Gabor Filter Design for Multiple Texture Segmentation, Optical Engineering, Vol. 35, no. 10, pp.2852-2863, October 1996.

9. Jain, A. K. and S. K. Bhattacharjee, Address block location on envelopes using Gabor filters. Pattern Recognition. 25(12): 1459-77, 1992.

10. P.P. Ohanian and R.C. Dubes, Performance evaluation for four classes of textural features, Pattern Recognition 25(8), pp. 819-833, 1992.

11. B. Huet and E.R. Hancock. Structural Indexing of Infra-Red images using Statistical Histogram Comparison, Third International Workshop on Image and Signal Processing (IWISP'96), Manchester (UK), pp. 4-7 Nov 1996. 